\title{
En guise de conclusion
}

Jules Maurin

\section{OpenEdition}

Journals

Édition électronique

URL : http://journals.openedition.org/trema/1899

DOI : 10.4000/trema.1899

ISSN : 2107-0997

\section{Éditeur}

Faculté d'Éducation de l'université de Montpellier

\section{Édition imprimée}

Date de publication : 1 décembre 1997

Pagination : 185-186

ISSN : 1167-315X

\section{Référence électronique}

Jules Maurin, «En guise de conclusion », Tréma [En ligne], 12-13| 1997, mis en ligne le 01 décembre 1997, consulté le 20 avril 2019. URL : http://journals.openedition.org/trema/1899 ; DOI : 10.4000/ trema.1899

Ce document a été généré automatiquement le 20 avril 2019

Trema 


\title{
En guise de conclusion
}

\author{
Jules Maurin
}

1 Les actes de ce colloque sur l'école rurale qui s'est tenue à Mende les 27 et 28 juin 1997 constituent la deuxième publication du Centre de Recherche sur l'École Rurale créé par convention entre quatre partenaires en 1995 : l'Université Paul Valéry-Montpellier III, l'Institut Universitaire de Formation des Maîtres de l'Académie de Montpellier, le Conseil Général du Département de la Lozère et l'association «Musécole du Gévaudan et des Cévennes ». La première publication, il y a deux ans, correspondait, de fait, au lancement du centre. Cette seconde parution en consacre et en pérennise l'existence. La revue Tréma dans laquelle sont publiées ces actes correspond bien par son esprit à celui de ce colloque.

Riche, ce colloque l'a été par la variété et la qualité des intervenants, tous universitaires confirmés venus à notre appel de la République Fédérale d'Allemagne, de Toulouse et de Montpellier bien sûr. Leur approche est différente selon qu'ils sont historiens ou linguistes; elles se complètent.

3 Six communications ont été consacrées aux aspects institutionnels de l'école rurale aux $\mathrm{XIX}^{\mathrm{e}}$ et $\mathrm{XX}^{\mathrm{e}}$ siècles présentant ici le cadre national et le rôle des politiques, et là des cas concrets départementaux, exemples choisis dans la variété du fonctionnement institutionnel de l'école rurale au niveau départemental. La réglementation en la matière, même rigide, admettait toujours un espace de liberté, d'initiative pour ceux qui voulaient bien s'y aventurer.

4 Six autres interventions traitent des mythologies scolaires et de la réception de l'enseignement c'est-à-dire du fond. Là encore, les aspects nationaux fondateurs des mythes et des traditions sont abordés sans que soient omis les exemples départementaux ou locaux toujours éclairants. Ainsi l'ensemble des aspects sont abordés et parfois dans leur contexte idéologique: école laïque et école libre catholique, gratuité de l'enseignement, question de la langue usuelle et du français, comportement locaux et familiaux.

5 Ces deux thèmes étaient vastes et il n'était pas possible de tout traiter. Le chantier reste ouvert. Mais des fenêtres sont maintenant pratiquées. En tout cas, cette pierre apportée à 
l'édifice d'une connaissance approfondie par les actes de ce colloque ne passera pas inaperçue.

6 Ce livre rendra service à tous ceux qui, aujourd'hui ou demain, par profession ou tout simplement par civisme, s'intéressent et s'intéresseront à l'école primaire et à son rôle éducatif et social. Il leur apportera, je crois, un minimum de profondeur historique qui leur permettra de mesurer combien l'école rurale, école de l'apprentissage des savoirs élémentaires est fondamentale pour l'insertion des enfants et des adolescents dans la vie et dans la société ; mais aussi et au-delà combien était important son rôle éducatif et civique. Cela devrait éviter les appréciations et jugements sommaires et trop souvent anachroniques sur ce que fut l'école de nos parents, grands-parents et arrières grandsparents. En tant que tel, ce livre suscitera des questions, déclenchera des interrogations et permettra donc d'avancer dans la découverte, dans l'approfondissement de nos connaissances sur l'école rurale.

7 Mais ce livre plaira aussi à tous ceux qui ont vécu ou entendu parler dans leur famille de cette école. Il provoquera l'émergence de souvenirs enfouis et partant, il devrait susciter des témoignages nombreux et spontanés sur ce qui fut naguère l'âge d'or de l'école rurale.

8 Ce colloque et cet ouvrage constituent donc pour les initiateurs de cette rencontre une étape dans la réalisation de l'un des objectifs du Centre de Recherche sur l'École Rurale de Mende, à savoir la constitution d'un fonds documentaire national original qui, à son tour, suscitera des recherches et nécessitera alors la confrontation des idées nouvelles émergentes lors d'une nouvelle rencontre.

9 C'est ainsi que, peu à peu, progressera la recherche en ce domaine. Ce colloque et les actes qui en sont issus n'ont pas d'autre but que d'y contribuer.

\section{RÉSUMÉS}

Non disponible

Not available

\section{AUTEUR}

JULES MAURIN

Université Paul Valéry de Montpellier 